\title{
Induced Quantitative Variation for Penicillin Titre in Clonal Populations of Aspergillus nidulans
}

\author{
By I. N. SIMPSON* AND C. E. CATEN \\ Department of Genetics, University of Birmingham, Birmingham B15 $2 T T$
}

(Received 19 June 1978)

\begin{abstract}
The variation in penicillin titre within populations of cultures of Aspergillus nidulans derived from untreated conidia and from conidia treated with ethyl methanesulphonate (EMS), near-ultraviolet light in the presence of 8-methoxypsoralen (8MOP) or $N$-methyl- $N^{\prime}$-nitro$N$-nitrosoguanidine (NTG), each at several dose levels, was determined. Both mutagentreated and untreated populations showed a continuous distribution of penicillin titres. The population mean titre of the mutagenized populations was decreased and the range of titres was increased relative to those of the control populations. No differences between sister cultures could be detected in three untreated populations, but nine out of ten populations derived from mutagenized conidia showed significant variation for penicillin titre. In general the magnitude of this induced variation increased with increasing dosage of the mutagen. Comparisons at fixed survival levels indicate that 8MOP mutagenesis is less effective for the induction of variation in penicillin titre than EMS or NTG mutagenesis. A statistical procedure was adopted to classify the survivors as unchanged cultures (' 0 '), titre-increasing mutants (' + ') or titre-decreasing mutants ('-'). The frequency of both ' + ' and ' - ' mutants increased following mutagenesis, with NTG being the most active of the three mutagens. Over all treatments, these two mutant classes were recovered with equal frequency. The frequency of ' + ' mutants was largely independent of mutagen dose, within the ranges used, and moderate treatments (around 10\% survival) gave as high or higher frequencies than more extreme doses. All three mutagens, and in particular NTG, produced morphological mutants. These contained an increased frequency of titre-decreasing mutants, but increases in titre appeared to be independent of changes in colony morphology. Estimates based on the observed frequencies of penicillin titre mutants suggest that several hundred genes are potentially capable of affecting this continuous variable.
\end{abstract}

\section{INTRODUCTION}

Successive cycles of mutation and selection have been widely used for the improvement of antibiotic yields in fungi and actinomycetes (e.g. Dulaney, 1953; Backus \& Stauffer, 1955; Elander et al., 1961). In this way large increases in productivity have been achieved through the stepwise accumulation of favourable mutations, many of which are individually of small effect (Alikhanian, 1970; Elander \& Espenshade, 1976). Consequently it would be predicted that the productivity of these improved strains would be determined by a polygenic system whose individual factors might interact in a non-additive manner. The present paper reports part of an investigation which was undertaken to test this prediction through genetic analysis of mutation selected strains. Previous attempts utilizing improved strains of Penicillium chrysogenum were frustrated by the lack of a true sexual cycle and by possible chromosomal damage produced by the repeated mutagenesis (Macdonald et al., 1964;

* Present address: Glaxo-Allenburys Research Ltd, Greenford, Middlesex UB6 0HE. 0022-1287/79/0000-8218 \$02.00 (C) 1979 SGM

Vol. 109, No. 2 was issued 13 December 1978 
Macdonald, 1968; Sermonti, 1969). To avoid these difficulties two precautions were taken. Firstly, the sexual species Aspergillus nidulans was employed; certain strains of this species produce a low level of an antibiotic which is indistinguishable from penicillin (Holt \& Macdonald, 1968a). Secondly, the improved strains were derived ab initio from a known translocation-free parent using only mutagens believed to cause little or no gross chromosomal damage.

In embarking upon a recurrent mutation and selection programme the choice of mutagen and dose level presents a practical problem. The mutagens used in strain improvement in the past appear to have been chosen largely on an empirical basis or to provide a contrast to a previous treatment (Backus \& Stauffer, 1955). More recently, test systems have been developed which detect major, qualitative mutations at several loci and so facilitate comparisons of mutagen efficiency (Lilly, 1965; Alderson \& Scazzocchio, 1967). However, antibiotic yield is a continuous variable dependent upon complex polygenic action (Alikhanian, 1970; Merrick \& Caten, 1975b) and the relevance of such test systems to the induction of quantitative genetic variation may be questioned. Direct studies of the effects of mutagens on antibiotic yields have been carried out by Alikhanian and co-workers (Alikhanian, 1962, 1970) and by Brown \& Elander (1966). Several generalizations have emerged from these studies: (i) mutagenic treatment increases the variation for antibiotic yield; (ii) the mean yield decreases following mutagenesis; (iii) the frequency of titre-increasing variants is maximized at intermediate levels of mutagen dose; (iv) the frequency of titre-decreasing variants is higher among morphologically changed than among morphologically normal survivors; (v) the optimum mutagen is strain dependent. The results from extensive investigations of induced quantitative variation in higher organisms, particularly higher plants, are in agreement with these conclusions (Brock, 1965; Borojević, 1966; Gregory, 1966).

Two mutagens were required for the mutation and selection programme. In choosing these, the requirement for minimum chromosomal damage was of prime importance and on this criterion three mutagens, $N$-methyl- $N^{\prime}$-nitro- $N$-nitrosoguanidine (NTG), ethyl methanesulphonate (EMS) and near-ultraviolet light in the presence of 8-methoxypsoralen (8MOP), were picked for initial comparison. The available evidence suggests that these act primarily as point mutagens and rarely induce chromosomal aberrations (Malling \& De Serres, 1968, 1970; Alderson \& Scott, 1970; Ball, 1973; Scott et al., 1976). This short list includes mutagens acting through each of the two major mutational pathways in Escherichia coli. Mutagenesis by $8 \mathrm{MOP}$ requires an error-prone pathway dependent upon both the $\operatorname{lex} A$ (formerly $\operatorname{exr} A$ ) and $\operatorname{rec} A$ genes and hence is active only on $\operatorname{lex} A^{+}$rec $A^{+}$strains. EMS and NTG, however, mutate strains carrying either lexA or recA mutations and hence must act through an alternative pathway (Bridges, 1976). We here report experiments designed to compare the efficiency of these three mutagens and to facilitate choice of the optimum mutational conditions for the strain selection programme. To this end, the induced quantitative variation in penicillin titre was estimated for each of the three mutagens at a range of dose levels.

\section{METHODS}

Strains. Two strains of Aspergillus nidulans, $7 / 142$ (biA1) and 7/151 (pabaAI;wA3), were used. These were both descended from the 'Glasgow' wild type, NRRL 194. Strain 7/142 was a subculture of FGSC 26 and carries no known translocations (Barratt et al., 1965). Strain 7/151 was derived from strain $7 / 148$ which also carries the $w A 3$ and pabaAl markers and was itself synthesized from known translocation-free strains. Strain $7 / 148$ was repeatedly backcrossed to $7 / 142$, a single $w$ paba progeny being selected in each generation. Strain $7 / 151$ was the third generation derivative of this backcrossing programme. As a result of their common ancestry and the repeated backcrossing, strains $7 / 142$ and $7 / 151$ should be isogenic, except for the three markers. Holt \& Macdonald $(1968 a, b)$ showed that $A$. nidulans NRRL 194 and its bi- 1 derivative (equivalent to FGSC 26) produced $6 \mathrm{u} \mathrm{ml}^{-1}$ of antibiotic indistinguishable from penicillin.

Strains in current use were stored on appropriately supplemented Czapek agar at $4{ }^{\circ} \mathrm{C}$; stock cultures were kept as dry conidia on silica gel at $4{ }^{\circ} \mathrm{C}$ (Roberts, 1969). 
Media. Czapek agar (CZ), supplemented with biotin $\left(1 \mu \mathrm{g} \mathrm{ml}^{-1}\right.$, final concentration) or $p$-aminobenzoic acid $\left(2 \mu \mathrm{g} \mathrm{ml}^{-1}\right.$, final concentration) as appropriate, was used as the basic medium for routine transfers, crossing etc. When plating conidial or ascospore suspensions, sodium deoxycholate (BDH) was added to the medium at $1.2 \mathrm{mg} \mathrm{ml}^{-1}$ (final concentration) to reduce colony size; this is denoted by $\mathrm{D}$. The conidial suspensions required for inoculating shake flasks or for mutagenic treatment were prepared from 5 or $6 \mathrm{~d}$-old slopes of $2 \%(\mathrm{w} / \mathrm{v})$ malt extract agar supplemented with $50 \mathrm{ml}$ complete medium supplement $\mathbf{l}^{-1}$. Complete medium supplement had the following composition: $2 \%(\mathrm{w} / \mathrm{v})$ Difco Bacto Casamino acids, $2 \%(\mathrm{w} / \mathrm{v})$ Difco Bacto peptone, $2 \%(\mathrm{w} / \mathrm{v})$ Difco Bacto yeast extract, $20 \%(\mathrm{v} / \mathrm{v})$ Aspergillus vitamin solution (Clowes \& Hayes, 1968), $0 \cdot 3 \%(\mathrm{w} / \mathrm{v})$ adenine. The composition of the fermentation medium for penicillin production and that of the bioassay medium were as given by Macdonald $e$ t al. (1963) and by Merrick \& Caten (1975b), respectively. The fermentation medium was dispensed in $40 \mathrm{ml}$ amounts into $250 \mathrm{ml}$ Erlenmeyer fiasks which were plugged with disposable foam bungs and autoclaved for $15 \mathrm{~min}$ at $100 \mathrm{kPa}$. Conidial suspensions were prepared in $0.02 \%(\mathrm{v} / \mathrm{v})$ Calsolene oil (ICI).

Mutagenesis. For treatment with $N$-methyl- $N^{\prime}$-nitro- $N$-nitrosoguanidine (NTG) the procedure of Clutterbuck \& Sinha (1966) was followed with a few modifications: $10 \mathrm{ml}$ conidial suspension $\left(10^{8}\right.$ spores $\left.\mathrm{ml}^{-1}\right)$ in $0.05 \mathrm{M}$-phosphate buffer pH 6.0 were pipetted into a $50 \mathrm{ml}$ conical flask, and $6 \mathrm{mg} \mathrm{NTG} \mathrm{(Ralph} \mathrm{H.} \mathrm{Emanuel,}$ Wembley, HA0 1PY) dissolved in $10 \mathrm{ml}$ buffer were added to start the treatment. Thus the final NTG concentration was $0.3 \mathrm{mg} \mathrm{ml}^{-1}$. During the mutagenic treatment, the conidial suspension was incubated in a shaking water bath at $37^{\circ} \mathrm{C}$. Samples $(1.0 \mathrm{ml})$ were removed at $10 \mathrm{~min}$ intervals and diluted in $5 \mathrm{ml} \mathrm{Calsolene}$ solution $(0.02 \%, \mathrm{v} / \mathrm{v})$. The treated conidia were washed twice by centrifugation and resuspension, then diluted appropriately and plated on supplemented CZD.

For treatment with ethyl methanesulphonate (EMS), $13.5 \mathrm{ml}$ conidial suspension $\left(10^{7}\right.$ spores $\left.\mathrm{ml}^{-1}\right)$ in $0.01 \mathrm{M}$-phosphate buffer $\mathrm{pH} 7.0$ were placed in a $25 \mathrm{ml}$ conical flask, and treatment was started by adding $1.5 \mathrm{ml}$ EMS (Sigma). The fiask was incubated as described above for NTG. Samples $(1.0 \mathrm{ml})$ were removed at appropriate intervals and the mutagen was diluted with $0.02 \%(\mathrm{v} / \mathrm{v})$ Calsolene solution by at least a factor of 100 before plating on supplemented CZD.

For treatment with near-ultraviolet light in the presence of 8-methoxypsoralen (8MOP), the procedure of Alderson \& Scott (1970) was followed: $1 \mathrm{mg} 8 \mathrm{MOP}$ (Xanthotoxin puriss, Koch-Light) dissolved in 0.5 ml $70 \%(\mathrm{v} / \mathrm{v})$ ethanol was added to $9.5 \mathrm{ml}$ conidial suspension $\left(10^{6}\right.$ spores $\left.\mathrm{ml}^{-1}\right)$, giving a final $8 \mathrm{MOP}$ concentration of $100 \mu \mathrm{g} \mathrm{ml}^{-1}$. After $5 \mathrm{~min}$ incubation at room temperature, the suspension was placed in an open glass Petri dish on a magnetic stirrer and irradiated with a Gallenkamp $125 \mathrm{~W}$ mercury discharge black lamp ( $95 \%$ output at $365 \mathrm{~nm}$ ) arranged $30 \mathrm{~cm}$ above the conidial suspension. At $5 \mathrm{~min}$ intervals, $0.1 \mathrm{ml}$ samples were removed, diluted appropriately and plated on supplemented CZD.

Strain $7 / 151$ was used for the EMS mutagenesis and strain $7 / 142$ for NTG and $8 \mathrm{MOP}$. Three different treatment times were used for NTG (expts 11 to 13) and EMS (expts 2 to 4 ) and four for 8MOP (expts 7 to 10) (Table 1). For all mutagenic treatments, untreated conidial suspensions were also plated on CZD to allow estimation of the survival levels of the treated suspensions (Table 1). Following mutagenesis, a random sample of 50 to 100 survivors was picked off in each experiment and transferred to $5 \times 5$ master plates ready for penicillin yield testing. These survivors were scored for any morphological changes induced by the mutagenic treatments and replicated on to unsupplemented $\mathrm{CZ}$ to check for reversion of the auxotrophic markers and contamination. Two samples of untreated single conidial descendants of $7 / 142$ (expts 5,6$)$ and one of 7/151 (expt 1) were similarly isolated to provide estimates of the spontaneous variation (Table 1).

Fermentation and penicillin yield testing. The procedure of Merrick \& Caten $(1975 a, b)$ was followed. In most experiments approximately 50 survivors (Table 1 ) were fermented, each in two replicate flasks. A single diluted broth sample was assayed from each flask, with the samples from the replicate flasks of each survivor assigned to different $8 \times 8$ assay plates. In these experiments assay plates were equivalent to blocks. Larger populations were tested in experiments 3 and 7 (Table 1). In experiment 7 three assay plates were divided into two blocks, each of one and a half plates, and the two replicates of each survivor were assigned to different blocks. Only a single flask was used for each of the 97 survivors in experiment 3 . In this case four assay plates were divided into two blocks and two broth samples were taken from each flask, one being plated in each block. Five replicate flasks of the parent strain $(7 / 142$ or $7 / 151)$ were incorporated as controls in all experiments involving mutagenized populations. Each assay plate included three wells of standard benzyl penicillin solutions (Glaxo) at $2.0,1.0$ and $0.5 \mathrm{u} \mathrm{ml}^{-1}$. The treated cultures, control cultures and standards were allocated to wells on the assay plates in a completely random manner. Each well was inoculated with $70 \mu \mathrm{l}$ of the appropriate solution using a microlitre pipette (Eppendorf, 2000 Hamburg 63, West Germany). An outer circuit of wells was cut in the assay plate around the $8 \times 8$ test wells and these were inoculated with $70 \mu \mathrm{l}$ of $0.01 \mathrm{M}$-phosphate buffer $\mathrm{pH} 7 \cdot 0$. These additional wells markedly reduced the tendency for the outer test wells to give inflated inhibition zones, i.e. the edge effect (Lees \& Tootill, 1955).

Statistical analysis. Inhibition zone diameters were converted to units of penicillin $\left(\mathrm{u} \mathrm{ml}^{-1}\right)$ using a Wang 600 Advanced Programmable Calculator (Merrick \& Caten, 1975a). To compensate for variation_between 
Table 1. Mutagenic treatments used and numbers of survivors assayed

\begin{tabular}{|c|c|c|c|c|}
\hline Expt no. & $\begin{array}{l}\text { Mutagenic } \\
\text { treatment* }\end{array}$ & $\begin{array}{c}\text { Parent } \\
\text { strain }\end{array}$ & $\begin{array}{c}\text { Survival } \\
(\%)\end{array}$ & $\begin{array}{c}\text { No. } \\
\text { assayed }\end{array}$ \\
\hline 1 & None & $7 / 151$ & 100 & 52 \\
\hline 2 & EMS, $30 \mathrm{~min}$ & $7 / 151$ & 29 & 50 \\
\hline 3 & EMS, $40 \mathrm{~min}$ & $7 / 151$ & 11 & 97 \\
\hline 4 & EMS, $60 \mathrm{~min}$ & $7 / 151$ & 0.04 & 50 \\
\hline 5 & None & $7 / 142$ & 100 & 49 \\
\hline 6 & None & $7 / 142$ & 100 & 33 \\
\hline 7 & $8 \mathrm{MOP}, 10 \mathrm{~min}$ & $7 / 142$ & 28 & 74 \\
\hline 8 & $8 \mathrm{MOP}, 15 \mathrm{~min}$ & $7 / 142$ & 10 & 46 \\
\hline 9 & $8 \mathrm{MOP}, 20 \mathrm{~min}$ & $7 / 142$ & 4 & 45 \\
\hline 10 & $8 \mathrm{MOP}, 60 \mathrm{~min}$ & $7 / 142$ & 0.09 & 50 \\
\hline 11 & NTG, $120 \min \dagger$ & $7 / 142$ & 65 & 49 \\
\hline 12 & NTG, $\quad 30 \mathrm{~min}$ & $7 / 142$ & 32 & 47 \\
\hline \multirow[t]{2}{*}{13} & NTG, $120 \mathrm{~min}$ & $7 / 142$ & 11 & 48 \\
\hline & & & & 690 \\
\hline
\end{tabular}

* EMS, Ethyl methanesulphonate; 8MOP, 8-methoxypsoralen plus near-ultraviolet light; NTG, $N$-methyl- $N^{\prime}$-nitro- $N$-nitrosoguanidine. See Methods for details.

$\dagger$ Final concentration of NTG was $0 \cdot 1 \mathrm{mg} \mathrm{ml}^{-1}$ rather than $0.3 \mathrm{mg} \mathrm{ml}^{-1}$.

fermentations run on different occasions, all population mean titres were standardized against the control strains (Merrick, 1975a). The standard analysis of variance of Merrick \& Caten (1975b) was applied to the population of strains derived from each experiment (the control strains were excluded from the analysis). For analysis, the observed titres were transformed to logarithms $\left(\log \mathrm{u} \mathrm{ml}^{-1}\right)$. This transformation removed the positive correlation observed between strain mean titre and replicate variance when titre was expressed as $\mathrm{u} \mathrm{ml}^{-1}$ (Merrick, 1975b). For ease of presentation, however, penicillin titre is shown as $\mathrm{u} \mathrm{ml}^{-1}$ in all figures and tables. The analysis provides estimates of the environmental variation $\left(\sigma_{E}{ }^{2}\right)$, the variation between assay blocks $\left(\sigma_{B}{ }^{2}\right)$ and the variation between sister strains $\left(\sigma_{\theta}{ }^{2}\right)$. In the present work, where sister strains are single conidial descendants of a common, homokaryotic parent, $\sigma_{G}{ }^{2}$ provides an estimate of the genetic variation in titre arising either spontaneously (expts $1,5,6$ ) or following mutagenesis (expts 2 to 4,7 to 10 , 11 to 13 ).

Definition of titre mutants. For the mutagen-treated populations (Table 1), survivors whose log titre exceeded or fell short of the untreated parental strain, fermented in the same experiment, by 1.96 standard deviations or more were defined as increasing ('+') or decreasing ('-') mutants, respectively. All survivors with $\log$ titres within the range parental $\log$ titre \pm 1.96 standard deviations were considered unchanged (' 0 ' strains). For the three untreated populations (expts $1,5,6$ ) ' + ', ' 0 ' and ' - ' strains were defined in a similar manner by their deviation from the mean log titre of the whole population.

The standard deviation used in the above definitions was calculated from the observed replicate variance $\left(\sigma_{E}{ }^{2}\right)$ from each experiment, and since the titre for each strain was based on two observations, the standard deviation was that appropriate to the mean of two observations, i.e. S.D. $=\sqrt{\frac{1}{2}} \sigma_{E}{ }^{2}$. With this procedure, assuming the errors of titre determination to be normally distributed and no spontaneous mutation, the expectation is that $2.5 \%$ of an untreated population would be classed as '+ ' variants, $2.5 \%$ as '- ' variants and the remaining $95 \%$ as unchanged (0). Significant deviations from this distribution would therefore indicate that the treatment is effective in producing titre mutants.

\section{RESULTS}

\section{Induced quantitative variation}

The distributions of penicillin titres among an untreated population (expt 1) and among survivors from EMS-treated populations of strain 7/151 at three survival levels (expts 2, $3,4)$ are shown in Fig. 1. Titres have been standardized against the control strains. Similar distributions were obtained with 8MOP- and NTG-treated populations, with both treated and untreated populations exhibiting continuous non-skewed distributions. The population mean penicillin titres were generally lower for mutagen-treated than for untreated populations (Table 2). That this reflects a consistent tendency for the mean titre to fall following 

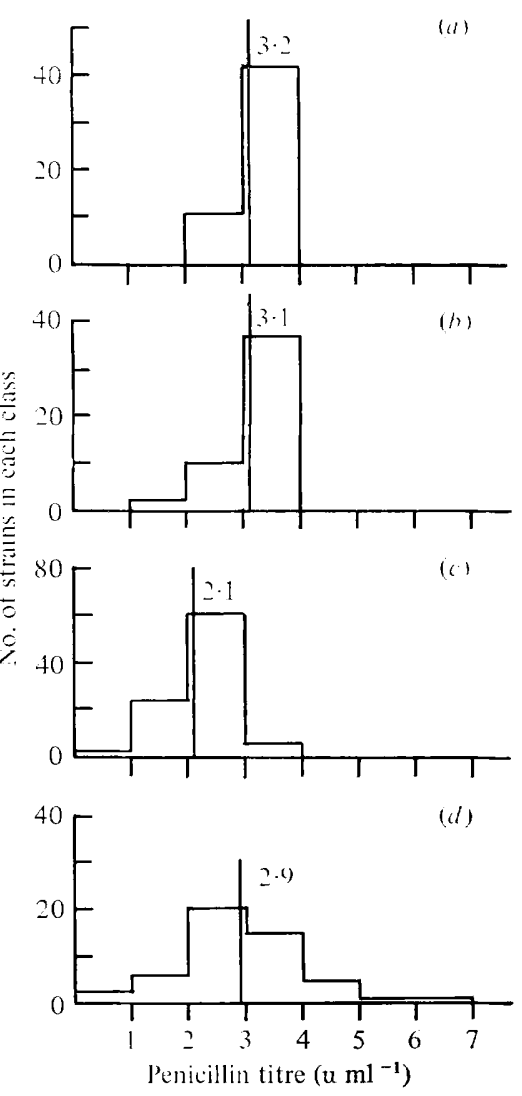

Fig. 1. Distribution of penicillin titre among clonal populations of strain $7 / 151$ derived without mutagenic treatment $(a)$ or after treatment with EMS to $29 \%$ survival $(b), 11 \%$ survival $(c)$ or $0.04 \%$ survival $(d)$. The bar indicates the population mean.

mutagenesis and is not an artifact resulting from the assay of the mutagen-treated and untreated populations in separate experiments is indicated by comparison of the mean of each treated population with the untreated parent assayed in the same experiment (control flasks). The mean log titre of the survivors was less than that of the control in nine of the ten mutagenized populations, although this difference was significant only for experiments 3 and 11. The range of penicillin titres was greater amongst mutagen-treated than untreated populations and in general increased with increasing mutagen dosage (Fig. 1, Table 2).

Estimates of $\sigma_{E}^{2}$ varied between 0.002 and 0.039 but these differences did not appear to be related either to the particular mutagen or to the dose level (Table 2). Thus there is no suggestion of induced instability within the survivors of mutagenic treatment. The high estimates obtained in experiments 7 and 10 can be attributed to the confounding of replicate observations with the difference between assay plates and to a shaker failure, respectively. Although frequently significant, the between assay blocks component of variation $\left(\sigma_{B}^{2}\right)$ was generally less than $\sigma_{E}{ }^{2}$. The aberrant estimate for experiment 10 was due to different fermentation conditions being used for the two blocks following the shaker failure.

No genetic component of variance $\left(\sigma_{G}{ }^{2}\right)$ was detected in any of the untreated populations (expts 1, 5, 6; Table 2). Hence, with the sample sizes used, spontaneous mutation was not a significant source of variation in penicillin titre. Following mutagenesis, however, significant levels of genetic variation were found in all cases except experiment 7, the lowest 
Table 2. Summary of analyses of variation in penicillin titre among control and mutagenized populations

\begin{tabular}{|c|c|c|c|c|c|c|c|}
\hline \multirow[b]{2}{*}{ Expt no. } & \multirow[b]{2}{*}{ Mutagen } & \multirow{2}{*}{$\begin{array}{c}\text { Survival } \\
(\%)\end{array}$} & \multicolumn{2}{|c|}{ Titre $\left(\mathrm{u} \mathrm{ml}^{-1}\right)$} & \multicolumn{3}{|c|}{ Variance components $\left(\log \mathrm{u} \mathrm{ml}^{-1}\right)$} \\
\hline & & & Mean & Range & $\sigma_{E}^{2}$ & $\sigma_{6}^{2}$ & $\sigma_{B}^{2}$ \\
\hline I & None & 100 & $3 \cdot 2$ & $2 \cdot 8-3 \cdot 7$ & 0.002 & 0.000 & $0.001 *$ \\
\hline 2 & EMS & 29 & $3 \cdot 1$ & $1.8-3.9$ & 0.003 & $0.003 *$ & $0.002 *$ \\
\hline 3 & EMS & 11 & $2 \cdot 1$ & $0 \cdot 0-4 \cdot 0$ & 0.002 & $0.089 *$ & $0.001 *$ \\
\hline 4 & EMS & 0.04 & $2 \cdot 9$ & $0 \cdot 3-6 \cdot 3$ & 0.019 & $0 \cdot 039 *$ & 0.000 \\
\hline 5 & None & 100 & $5 \cdot 8$ & $3 \cdot 3-8 \cdot 4$ & 0.019 & $0 \cdot 000$ & 0.000 \\
\hline 6 & None & 100 & $5 \cdot 8$ & $4 \cdot 4-7 \cdot 5$ & 0.009 & 0.001 & $0.002 *$ \\
\hline 7 & $8 \mathrm{MOP}$ & 28 & $4 \cdot 7$ & $2 \cdot 0-9.9$ & 0.037 & 0.005 & $0.004 *$ \\
\hline 8 & $8 \mathrm{MOP}$ & 10 & $6 \cdot 4$ & $3 \cdot 6-12 \cdot 2$ & 0.004 & $0.005^{*}$ & $0.003 *$ \\
\hline 9 & $8 \mathrm{MOP}$ & 4 & $5 \cdot 1$ & $1 \cdot 6-9 \cdot 8$ & 0.004 & $0.008^{*}$ & 0.000 \\
\hline 10 & $8 \mathrm{MOP}$ & 0.09 & $4 \cdot 8$ & $1 \cdot 0-9 \cdot 8$ & C 039 & $0.022 *$ & $0 \cdot 100^{*}$ \\
\hline 11 & NTG & 65 & $4 \cdot 5$ & $2 \cdot 0-8 \cdot 0$ & 0.005 & $0.016^{*}$ & $0.002 *$ \\
\hline 12 & NTG & 32 & $6 \cdot 3$ & $3 \cdot 9-13 \cdot 0$ & 0.010 & $0.005^{*}$ & $0.012 *$ \\
\hline 13 & NTG & 11 & $5 \cdot 0$ & $0.4-8.9$ & 0.008 & $0.040^{*}$ & $0.005 *$ \\
\hline
\end{tabular}

* Significantly different from zero, $P<0 \cdot 01$.
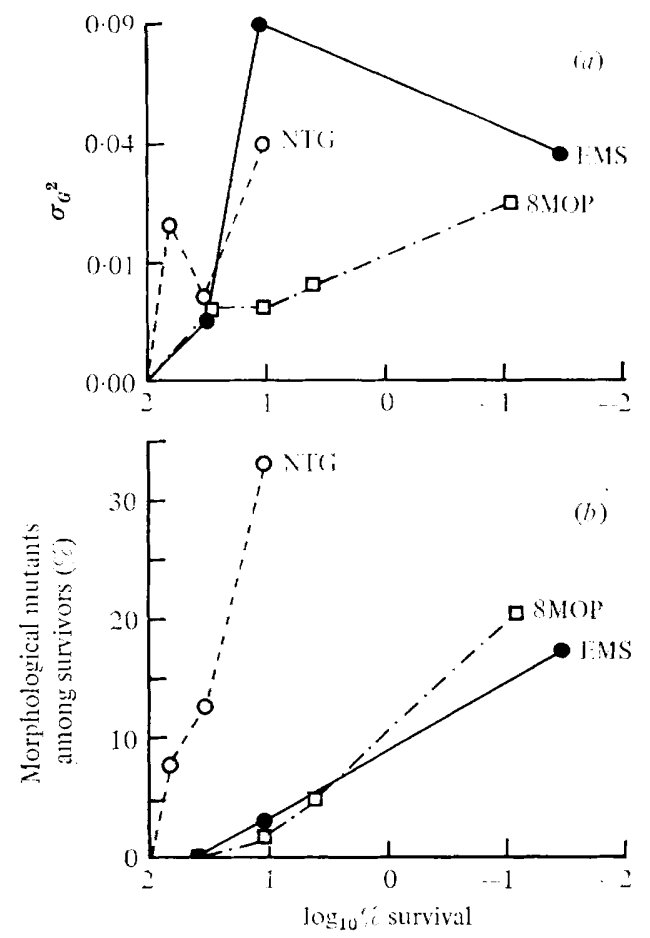

Fig. 2. The relationship between mutagen dose (expressed as $\log \%$ survival) and $(a)$ the genetic variation between sister cultures $\left(\sigma_{G}{ }^{2}\right)$ or $(b)$ the frequency of morphological mutants, for three mutagens.

$8 \mathrm{MOP}$ treatment (Table 2). Where significant, the estimates of $\sigma_{G}{ }^{2}$ were generally greater than those of $\sigma_{E}^{2}$ and ranged from 0.003 to 0.089 . This variation in the magnitude of the induced genetic variation is related both to the different mutagens and to the dose levels (Fig. 2a). Comparisons between mutagens at comparable survivals suggest that $8 \mathrm{MOP}$ is less efficient than NTG or EMS. The effect of dose is more striking, the estimate of $\sigma_{\sigma}{ }^{2}$ increasing with decreasing survival for all three mutagens. 


\section{Frequency of titre mutants}

The estimate of the genetic variance used above incorporates variation due to both titre increasing ('+') and decreasing ('-') mutations. However, only the former are of interest in strain improvement programmes, and since the variance may hide preferential induction of one or the other mutant type, conclusions arrived at only from consideration of this parameter may be misleading. Alikhanian $(1962,1970)$ avoided this problem by using the frequency of ' + ' and '-' titre variants to compare mutagenic treatments in Actinomyces spp. However, the recognition of these variants presents a problem since the distribution of titre among survivors is continuous (Fig. 1), and therefore it is necessary to adopt statistical definitions. The definitions used here have been described in Methods.

The distribution of strains in the three titre classes for each experiment is shown in Table 3. With titre mutants defined relative to the control strain, experiments 3 and 11 gave frequencies of variants (70\% and $45 \%$, respectively) which were much higher than those of the other experiments and higher than expected from the moderate dose levels involved. Furthermore, all the variants were of the " -' type. Definition of mutants relative to the controls has the result that any chance fluctuation in the control titre will greatly influence the apparent number of mutants. The control titres in experiments 3 and 11 were markedly greater than the population mean titre and if this were due to chance it would account both for the apparent high frequency of variants and their unidirectional nature. To overcome this problem the survivors in these two experiments were reclassified into titre classes on the basis of their deviation from the population mean, i.e. by the method used for the untreated populations. This procedure gave classifications which were more consistent with the other experiments in regard to both the overall frequency of variants and their distribution. It is these classifications which are given for experiments 3 and 11 in Table 3 and used for subsequent interpretation of the data. The difficulties encountered in these two experiments emphasize the need for a high level of replication of the control strain(s).

The untreated populations (expts 1,5,6) gave close approximations to the frequencies expected with uniform, normally distributed populations, in agreement with the absence of genetic differences within the populations (Table 3 ). In all cases the frequency of titre variants increased following mutagenesis. Of the survivors from mutagenic treatment $18.9 \%$ were classified as mutant compared with $3.0 \%$ of the untreated single conidial isolates (Table 3). In general the frequency of mutants increased with decreasing survival for each mutagen, in line with the trends previously observed in $\sigma_{\theta}{ }^{2}$ (Fig. $2 a$ ). Pooled over all experiments, the frequencies of ' + ' and ' - ' mutants were similar and this also applied to most individual experiments, although in a few instances there appeared to be an excess of '+' (expt 3) or '-' (expt 2) variants (Table 3). Of the three mutagens, NTG gave the highest frequencies both for total and ' + ' mutants, while there was little difference between EMS and $8 \mathrm{MOP}$. As regards mutagen dose and the induction of ' + ' mutants, maximum frequencies were given by EMS at $0 \cdot 04 \%$ survival, by 8 MOP at $4 \%$ survival and NTG at $65 \%$ survival, suggesting no consistent relationship (Table 3 ).

\section{Morphological mutants and titre variation}

No morphological mutants were found within the untreated populations from either strain. All of the mutagenic treatments generated morphological mutants. However, these were present at such low frequencies in the lowest EMS and 8MOP treatments (expts 2 and 7 , respectively) that none were included in the samples taken for penicillin assay (Table 4). As expected, the frequency of morphological mutants increased with increasing dose for each mutagen and there was no suggestion of maximum frequencies within the dose ranges used (Fig. 2b). NTG was particularly effective in producing morphological mutants. 
Table 3. Frequency of penicillin titre variants among untreated and mutagen-treated populations

\begin{tabular}{|c|c|c|c|c|c|}
\hline \multirow[b]{2}{*}{ Expt no. } & \multirow[b]{2}{*}{ Mutagen } & \multirow{2}{*}{$\begin{array}{c}\text { Survival } \\
(\%)\end{array}$} & \multicolumn{3}{|c|}{ No. $(\%)$ of variants in titre class* } \\
\hline & & & + & 0 & - \\
\hline 1 & None & 100 & $1(2)$ & $50(96)$ & $1(2)$ \\
\hline 2 & EMS & 29 & $1(2)$ & $43(86)$ & $6(12)$ \\
\hline 3 & EMS & 11 & $9(9)$ & $84(87)$ & $4(4)$ \\
\hline 4 & EMS & 0.04 & $5(10)$ & $38(76)$ & $7(14)$ \\
\hline 5 & None & 100 & $1(2)$ & $48(98)$ & $0(0)$ \\
\hline 6 & None & 100 & $1(3)$ & $32(97)$ & $0(0)$ \\
\hline 7 & $8 \mathrm{MOP}$ & 28 & $5(7)$ & $67(90)$ & $2(3)$ \\
\hline 8 & $8 \mathrm{MOP}$ & 10 & $3(7)$ & $40(86)$ & $3(7)$ \\
\hline 9 & $8 \mathrm{MOP}$ & 4 & $4(9)$ & $39(87)$ & $2(4)$ \\
\hline 10 & 8MOP & 0.09 & $1(2)$ & $42(84)$ & $7(14)$ \\
\hline 11 & NTG & 65 & $9(18)$ & $32(66)$ & $8(16)$ \\
\hline 12 & NTG & 32 & $5(11)$ & $39(83)$ & $3(6)$ \\
\hline 13 & NTG & 11 & $7(14)$ & $27(57)$ & $14(29)$ \\
\hline \multicolumn{2}{|c|}{ Total, all treatments } & & $52(7 \cdot 5)$ & $581(84 \cdot 2)$ & $57(8 \cdot 3)$ \\
\hline \multicolumn{2}{|c|}{ Total, untreated } & & $3(2 \cdot 2)$ & $130(97 \cdot 0)$ & $1(0 \cdot 8)$ \\
\hline \multicolumn{2}{|c|}{ Total, mutagen-treated } & & $49(8 \cdot 8)$ & $451(81 \cdot 1)$ & $56(10 \cdot 1)$ \\
\hline
\end{tabular}

Table 4. Penicillin titre class of morphologically changed survivors

\begin{tabular}{|c|c|c|c|c|c|c|}
\hline \multirow[b]{2}{*}{ Expt no. } & \multirow[b]{2}{*}{ Mutagen } & \multirow{2}{*}{$\begin{array}{c}\text { Survival } \\
(\%)\end{array}$} & \multirow{2}{*}{$\begin{array}{l}\text { No. }(\%) \text { of } \\
\text { morphological } \\
\text { mutants }\end{array}$} & \multicolumn{3}{|c|}{ No. in titre class* } \\
\hline & & & & + & 0 & - \\
\hline 1 & None & 100 & $0(0)$ & $\mathbf{0}$ & 0 & 0 \\
\hline 2 & EMS & 29 & $0(0)$ & 0 & 0 & 0 \\
\hline 3 & EMS & 11 & $3(3)$ & 2 & 0 & 1 \\
\hline 4 & EMS & $0 \cdot 04$ & $9(18)$ & 1 & 6 & 2 \\
\hline 5 & None & 100 & $0(0)$ & 0 & 0 & 0 \\
\hline 6 & None & 100 & $0(0)$ & 0 & $\mathbf{0}$ & 0 \\
\hline 7 & $8 \mathrm{MOP}$ & 28 & $0(0)$ & 0 & 0 & 0 \\
\hline 8 & 8MOP & 10 & $1(2)$ & 0 & 1 & 0 \\
\hline 9 & $8 \mathrm{MOP}$ & 4 & $2(4)$ & 1 & 1 & 0 \\
\hline 10 & 8MOP & 0.09 & $10(21)$ & 1 & 5 & 4 \\
\hline 11 & NTG & 65 & $3(6)$ & 0 & 1 & 2 \\
\hline 12 & NTG & 32 & $6(13)$ & 1 & 4 & 1 \\
\hline \multirow[t]{2}{*}{13} & NTG & 11 & $16(33)$ & 1 & 11 & 4 \\
\hline & & Total & 50 & $7(14 \%)$ & $29(58 \%)$ & $14(28 \%)$ \\
\hline
\end{tabular}

* See Methods for definition of titre classes.

Table 5. Penicillin titre class of morphologically changed and morphologically unchanged survivors from eight mutagenic treatments*

No. $(\%)$ in titre class $\dagger$

Morphologically changed

Morphologically unchanged

$$
\text { Total }
$$

$\begin{array}{rrrr}+ & 0 & - & \text { Total } \\ 7(14 \cdot 0) & 29(58 \cdot 0) & 14(28 \cdot 0) & 50 \\ 36(9 \cdot 4) & 312(81 \cdot 7) & 34(8 \cdot 9) & 382 \\ 43(10 \cdot 0) & 341(78 \cdot 9) & 48(11 \cdot 1) & 432\end{array}$

* Only those treatments which produced morphological mutants are included, i.e. experiments $3,4,8$ to 13 (Table 4).

$\dagger$ See Methods for definition of titre classes. 
Survivors were selected at random for penicillin assay and hence morphologically both wild-type and mutant strains were included in the population fermented. The morphological mutants (50 altogether) included strains classified as ' + ' and ' - ' with respect to titre (Table 4). Considering those eight treatments where morphological mutants were included in the sample assayed (Table 4), the morphologically changed survivors showed a significantly $\left(\chi_{[1]}^{2}=14.90, P=<0.001\right)$ higher frequency of titre mutants $(42 \%)$ than the morphologically unchanged survivors $(18 \%)$ (Table 5). This higher frequency, however, was entirely due to an increase in ' - ' mutants, there being no significant difference in the frequency of ' + ' mutants between the morphologically changed and unchanged survivors $\left(\chi_{[1]}^{2}=1 \cdot 03, P=0.5\right.$ to $\left.0 \cdot 3\right)$.

\section{DISCUSSION}

All three mutagens were effective in inducing variation in penicillin titre among clonal populations of $A$. nidulans and, in general, the magnitude of the induced variation increased with increasing dose of the mutagen (Fig. $2 a$ ). The increased variance of mutagen-treated populations was attributable to the induction of both increasing ('+') and decreasing ('-') mutants, these two types appearing in approximately equal frequency (Table 3 ). However, the observed tendency for the population mean to decrease following mutagenesis suggests that on average the '-' mutants had a greater effect. For example, three mutants were recovered with titres less than $10 \%$ that of their parent but none with a corresponding proportional increase. These three extreme variants correspond to major 'penicillinless' mutants (npe) as defined by Edwards et al. (1974). Dose-dependent increases in the variance of quantitative characters following mutagenesis have been reported in micro-organisms (Alikhanian, 1962, 1970; Brown \& Elander, 1966) and higher plants (Gaul, 1965). Brown \& Elander (1966) found that the mean potency relative to untreated controls decreased following mutagenesis and that the magnitude of this decrease was linearly related to the mutagen dose. A similar decrease has been frequently observed with higher plants (Gregory, 1955; Gaul, 1965; Brock, 1965) and variously attributed to preferential induction of '- - mutants (Sakai \& Suzuki, 1964; Gaul, 1965), or, as here, to '-' mutants having an average greater effect (Gregory, 1966). From extensive investigations of the effects of mutagenic treatment on variation in antibiotic production in Actinomyces spp., Alikhanian (1962) concluded that the relative frequency of ' + ' and ' - ' variants was dependent upon the parental strain, the mutagen and the dose level. In general the proportion of '+' variants was highest when the treated strain had not been previously selected and at low to moderate doses. The equal frequency of these two types of variant in the present study, where wild type strains were used as parents and high dose rates were not employed, is consistent with Alikhanian's findings.

The higher range of survival levels used with NTG makes comparison between the mutagens difficult. However, the two lowest survival levels for NTG (32 and $11 \%$ ) are similar to the two highest for EMS (29 and $11 \%$ ) and 8MOP (28 and $10 \%)$. At both levels NTG was the most active and produced the highest frequencies of titre and morphological mutants. This high mutation to lethality ratio of NTG was particularly apparent with the morphological mutants (Fig. $2 b$ ) and is widely appreciated, although the origin of the effect is not clear (Bridges, 1976). 8MOP, on the other hand, was the least efficient of the mutagens when assessed by the amount of induced variation for penicillin titre (Fig. $2 a$ ) but was similar to EMS in terms of the frequency of titre and morphological mutants (Table 3, Fig. $2 b$ ). Reproducible survival levels could not be obtained with NTG despite standardization of the concentration and incubation conditions. This variability would make it difficult to use this mutagen for successive mutagenic treatments where a constant effective dose is desired.

Extensive investigations involving forward and reverse mutational systems and physical and chemical mutagens have shown that the frequency of mutants among survivors generally 
increases with increasing dose and only reaches a plateau at very low survival levels (Stapleton et al., 1952; Esser \& Kuenen, 1967). This expected dose-response relationship is clearly shown by the morphological mutants (Fig. $2 b$ ) and to a lesser extent by the titre-decreasing mutants (Table 3). The frequency of titre-increasing mutants, however, appears to be independent of dose over much of the range examined and may even decline at the higher doses (e.g. the 8 MOP treatments, Table 3). With all three mutagens, moderate treatments giving around $10 \%$ survival produced frequencies of ' + ' mutants which were not significantly different from the maximum observed levels (Table 3 ). This suggests that intermediate doses are the most efficient for the induction, in the Glasgow strain of $A$. nidulans, of variants producing higher yields of penicillin. This conclusion is in agreement with results for Actinomyces spp. (Alikhanian, 1962) and with antibiotic AD-464 (Brown \& Elander, 1966 ) and with experience from strain improvement programmes (Elander \& Espenshade, 1976), leading to the generalization that intermediate doses (30 to $5 \%$ survival) are most efficient for the induction of quantitatively improved variants. With quantitative characters such as antibiotic yield many genes are involved (Merrick \& Caten, $1975 b$; Caten \& Jinks, 1976) and at high doses the incorporation of advantageous mutations may be masked by simultaneous deleterious ones. Most mutational test systems, on the other hand, require a change at one of a limited number of specific loci and there is little chance of interference from other simultaneous mutations, even with the high mutation frequencies obtained at high doses.

Examination of the relationship between changes in colony morphology and changes in titre indicated that the morphological mutants included an excess of ' -' mutants (Table 5). This association probably reflects the negative pleiotropic effect of many major mutations upon penicillin titre (Macdonald et al., 1963). Increases in penicillin titre, on the other hand, were independent of morphological changes (Table 5) indicating no pleiotropic effects. Likewise, Alikhanian (1962) found that morphological mutants of Actinomyces spp. contained more '-' but fewer ' + ' variants than did survivors with unchanged morphology, while Brown \& Elander (1966) concluded that the selection of morphologically 'normal' as opposed to 'abnormal' survivors increased the probability of detecting an enhanced variant 10 -fold. Thus three studies involving different organisms and antibiotics indicate that strain improvement through mutation and selection would not be facilitated, and may even be hindered, by the selection of morphologically changed survivors. This conclusion is, however, at variance with the observation that many strains of Penicillium chrysogenum, Streptomyces rimosus and $A$. nidulans selected as showing major qualitative increases in antibiotic productivity were morphologically changed (Backus \& Stauffer, 1955; Demain, 1973; Ditchburn et al., 1976).

The high frequency of recovery of penicillin titre variants (Table 3) suggests that these originate through mutation at any of a large number of loci. Estimates of the number of loci affecting sporulation in bacteria and fungi have been obtained from comparisons of the frequency of sporulation mutants with that of mutants induced by the same mutagenic treatments in test systems involving known numbers of loci (Balassa, 1969; Martinelli \& Clutterbuck, 1971). The mutagenicity in such test systems of the precise treatments used in the present work was not determined alongside the assays for titre variation; however, data are available in the literature for comparable treatments of all three mutagens (Alderson \& Clark, 1966; Alderson \& Hartley, 1969; Alderson \& Scott, 1970; Scott \& Alderson, 1971; Martinelli \& Clutterbuck, 1971). Following the method of Balassa (1969), the number of loci which can affect penicillin titre is given by:

\section{$\frac{\text { frequency of titre mutants }}{\text { frequency of mutants in the test system }} \times$ number of loci in the test system}

The frequency of titre mutants was taken as the sum of $\%$ ' + ' and \% ' - ' variants 
(Table 3 ) less $5 \%$ for the tails of the untreated population. Estimates obtained in this way varied from 75 to 2250 with a mean of 982 , depending upon the particular mutagenic treatment used. While these estimates involve many assumptions and must be treated with caution, they do emphasize that penicillin titre in $A$. nidulans is potentially subject to polygenic determination. It is not surprising therefore that the variation in penicillin titre among wild isolates of this species is inherited in a complex manner (Merrick \& Caten, $1975 b)$.

We thank Glaxo Research Laboratories Ltd, Sefton Park, Stoke Poges, Buckinghamshire, for the provision of equipment and materials and the Glaxo staff for their guidance and encouragement. We are particularly grateful to $\mathrm{Dr} \mathrm{C}$. Ball for helpful advice in preparing this paper. One of us (I.N.S.) gratefully acknowledges a Science Research Council CASE Studentship.

\section{REFERENCES}

Alderson, T. \& Clark, A. M. (1966). Interlocus specificity for chemical mutagens in Aspergillus nidulans. Nature, London 210, 593-595.

Alderson, T. \& Hartley, M. J. (1969). Specificity for spontaneous and induced forward mutation at several gene loci in Aspergillus nidulans. Mutation Research 8, 255-264.

Alderson, T. \& Scazzocchio, C. (1967). A system for the study of interlocus specificity for both forward and reverse mutation in at least eight gene loci in Aspergillus nidulans. Mutation Research 4, 567-577.

Alderson, T. \& Scotr, B. R. (1970). The photosensitizing effect of 8-methoxypsoralen on the inactivation and mutation of Aspergillus conidia by near ultraviolet light. Mutation Research 9, 569-578.

Alikhanian, S. I. (1962). Induced mutagenesis in the selection of microorganisms. Advances in Applied Microbiology 4, 1-50.

Alikhanian, S. I. (1970). Applied aspects of microbial genetics. Current Topics in Microbiology and Immunology 53, 91-148.

Backus, M. P. \& Stauffer, J. F. (1955). The production and selection of a family of strains in Penicillium chrysogenum. Mycologia 47, 429-463.

Balassa, G. (1969). Biochemical genetics of bacterial sporulation. I. Unidirectional pleiotropic interactions among genes controlling sporulation in Bacillus subtilis. Molecular and General Genetics 104, 73-103.

BALL, C. (1973). Improvement of penicillin productivity in Penicillium chrysogenum by recombination. In Genetics of Industrial Microorganisms, pp. 227-237. Edited by Z. Vaněk, Z. Hoštálek \& J. Cudlín. Prague: Academia.

Barratt, R. W., Johnson, G. B. \& Ogata, W. N. (1965). Wild type and mutant stocks of Aspergillus nidulans. Genetics 52, 233-246.

Borojević, K. (1966). Studies on radiation induced mutations in quantitative characters of wheat (Triticum vulgare). In Mutations in Plant Breeding, pp. 15-38. Vienna: International Atomic Energy Agency.

BridGes, B. A. (1976). Mutation induction. In Second International Symposium on the Genetics of Industrial Microorganisms, pp. 7-28. Edited by K. D. Macdonald. London: Academic Press.

Brock, R. D. (1965). Induced mutations affecting quantitative characters. In The Use of Induced Mutations in Plant Breeding, pp. 451-464. Supplement to Radiation Botany 5. Oxford: Pergamon Press.

Brown, W. F. \& Elander, R. P. (1966). Some biometric considerations in an applied antibiotic AD-464 strain development program. Developments in Industrial Microbiology 8, 114-123.

CATEN, C. E. \& Jinks, J. L. (1976). Quantitative genetics. In Second International Symposium on the Genetics of Industrial Microorganisms, pp. 93111. Edited by K.D. Macdonald. London: Academic Press.

Clowes, R. C. \& Hayes, W. (1968). Experiments in Microbial Genetics, p. 191. Oxford and Edinburgh: Blackwell Scientific Publications.

Clutterbuck, A. J. \& Sinha, U. K. (1966). NMethyl- $N^{\prime}$-nitro- $N$-nitrosoguanidine (NTG) as a mutagen for Aspergillus nidulans. Aspergillus Newsletter 7, 12-13.

DemaIN, A. L. (1973). Mutation and the production of secondary metabolites. Advances in Applied Microbiology 16, 177-202.

Ditchburn, P., Holt, G. \& Macdonald, K. D. (1976). The genetic location of mutations increasing penicillin yield in Aspergillus nidulans. In Second International Symposium on the Genetics of Industrial Microorganisms, pp. 213-227. Edited by K. D. Macdonald. London: Academic Press.

Dulaney, E. L. (1953). Observations on Streptomyces griseus VI. Further studies on strain selection for improved streptomycin production. Mycologia 45, 480-487.

Edwards, G. F. St. L., Holt, G. \& Macdonald, K. D. (1974). Mutants of Aspergillus nidulans impaired in penicillin biosynthesis. Journal of General Microbiology 84, 420-422.

Elander, R. P. \& Espenshade, M. A. (1976). The role of microbial genetics in industrial microbiology. In Industrial Microbiology, pp. 192-256. Edited by B. M. Miller \& W. Litsky. New York: McGraw-Hill. 
Elander, R. P., Stauffer, J. F. \& Backus, M. P. (1961). Antibiotic production by various species and varieties of Emericellopsis and Cephalosporium. Antimicrobial Agents Annual 1960, 91102.

ESSER, K. \& KuENEN, R. (1967). Genetics of Fungi. New York: Springer Verlag.

Gaul, H. (1965). The concept of macro- and micromutations and results on induced micro-mutations in barley. In The Use of Induced Mutations in Plant Breeding, pp. 407-428. Supplement to Radiation Botany 5. Oxford: Pergamon Press.

GREGORY, W. C. (1955). X-ray breeding of peanuts (Arachis hypogaea L.). Agronomy Journal 47, 396-399.

GREGORY, W. C. (1966). Mutation breeding. In Plant Breeding, pp. 189-218. Edited by K. J. Frey. Ames: Iowa State University Press.

Holt, G. \& Macdonald, K. D. (1968a). Penicillin production and its mode of inheritance in Aspergillus nidulans. Antonie van Leeuwenhoek 34, 409-416.

Holt, G. \& MACDonald, K. D. (1968b). Isolation of strains with increased penicillin yield after hybridisation in Aspergillus nidulans. Nature, London 219, 636-637.

LeEs, K. A. \& Tootill, J. P. R. (1955). Microbiological assay on large plates. 1. General considerations with particular reference to routine assay. Analyst 80, 95-110.

LILLY, L. J. (1965). An investigation of the suitability of suppressors of meth 1 in Aspergillus nidulans for the study of induced and spontaneous mutations. Mutation Research 2, 192-195.

Macdonald, K.D. (1968). The persistence of parental genome segregation in Penicillium chrysogenum after nitrogen mustard treatment. Mutation Research 5, 302-305.

Macdonald, K. D., Hutchinson, J. M. \& Gillett, W. A. (1963). Isolation of auxotrophs of Penicillium chrysogenum and their penicillin yields. Journal of General Microbiology 33, 365-374.

Macdonald, K. D., Hutchinson, J. M. \& Gillett, W. A. (1964). Properties of heterozygous diploids between strains of Penicillium chrysogenum selected for high penicillin yield. Antonie van Leeuwenhoek 30, 209-224.

Malling, H. V. \& De Serres, H. J. (1968). Identification of genetic alteration induced by ethyl methane sulphonate in Neurospora crassa. Mutation Research 6, 181-193.
Malling, H. V. \& De Serres, F. J. (1970). Genetic effects of $N$-methyl- $N$-'nitro- $N$-nitrosoguanidine in Neurospora crassa. Molecular and General Genetics 106, 195-207.

Martinelli, S. D. \& Clutterbuck, A. J. (1971). A quantitative survey of conidiation mutants in Aspergillus nidulans. Journal of General Microbiology 69, 261-268.

MerricK, M. J. (1975a). Hybridization and selection for increased penicillin titre in wild-type isolates of Aspergillus nidulans. Journal of General Microbiology 91, 278-286.

MerRICK, M. J. (1975b). The inheritance of penicillin titre in crosses between lines of Aspergillus nidulans selected for increased productivity. Journal of General Microbiology 91, 287-294.

MerricK, M. J. \& CATEN, C. E. (1975a). The design of fermentation and biological assay procedures for assessment of penicillin production in populations of Aspergillus nidulans. Journal of Applied Bacteriology 38, 121-131.

Merrick, M. J. \& Caten, C. E. (1975b). The inheritance of penicillin titre in wild-type isolates of Aspergillus nidulans. Journal of General Microbiology 86, 283-293.

RoBERTS, C. (1969). Silica gel stock cultures of Aspergillus nidulans. Aspergillus Newsletter 10, 29.

SaKaI, K. \& SuzuKI, A. (1964). Induced mutation and pleiotropy of genes responsible for quantitative characters in rice. Radiation Botany 4, 141151.

ScotT, B. R. \& Alderson, T. (1971). The random (non-specific) forward mutational response of gene loci in Aspergillus conidia after photosensitisation to near ultraviolet light $(365 \mathrm{~nm})$ by 8-methoxypsoralen. Mutation Research 12, 29-34.

Scott, B. R., PATHAK, M. A. \& MohN, G. R. (1976). Molecular and genetic basis of furocoumarin reactions. Mutation Research 39, 29-34.

SERMONTI, G. (1969). Genetics of Antibiotic-producing Microorganisms. London: Wiley Interscience.

Stapleton, G. E., Hollaender, A. \& Martin, F. L. (1952). Mechanism of lethal and mutagenic action of ionizing radiation on Aspergillus terreus. I. Relationship of relative biological efficiency to ion density. Journal of Cellular and Comparative Physiology 39, suppl. 1, 87-100. 